\title{
DESIGN OF THE OPTICAL COMMUNICATION SYSTEM FOR THE ASTEROID IMPACT MISSION
}

\author{
C. Heese ${ }^{1}$, Z. Sodnik ${ }^{1}$, I. Carnelli ${ }^{2}$ \\ ${ }^{1}$ ESA-ESTEC, The Netherlands. ${ }^{2}$ ESA-HQ, France
}

\section{INTRODUCTION}

The Asteroid Impact Mission (AIM) is part of the joint Asteroid Impact \& Deflection Assessment (AIDA) project of ESA, DLR, Observatoire de la Côte d'Azur, NASA, and Johns Hopkins University Applied Physics Laboratory (JHU/APL). The intention of AIDA is to send two spacecraft to the binary asteroid 65803 Didymos (1996 GT):

- $\quad$ An asteroid impactor - the NASA/APL Double Asteroid Redirection Test (DART) mission led by JHU/APL.

- An asteroid rendezvous and observation spacecraft (AIM) led by ESA.

AIM is a small, low-cost technology mission of opportunity to explore and demonstrate technologies for future deep-space missions, while addressing planetary defense objectives and performing asteroid scientific investigations on the binary asteroid Didymos (1996 GT). The asteroid's larger component Didymain has a diameter of 800 meters and Didymoon of 150 meters and their separation is about $1.1 \mathrm{~km}$. The main mission objectives are to determine the binary asteroid's orbital and rotation dynamics as well as the mass, geophysical properties and surface and subsurface structure, both before and after the DART impact. In addition, AIM is to demonstrate novel spacecraft technologies and operations to advance science and exploration missions, particularly optical communications and the use of an RF inter-satellite link with CubeSats in interplanetary space. If approved it will be ESA's first mission to a binary asteroid.

The AIM mission has five primary research objectives to determine:

1. The binary asteroid's physical properties like size, mass, shape and density. This will be realized by creating a shape model based on inputs from the Visual Imaging System (VIS) and a laser altimeter (OPTEL-D).

2. The binary asteroid's orbital and rotation dynamics, using as a minimum a Visual Imaging System (VIS).

3. The geophysical surface properties. The VIS instrument is used for detecting surface features and the Thermal InfraRed Imager (TIRI) for surface roughness. In addition the subsurface structure will be probed with the High-Frequency Radar (HFR) and the accelerometers on the MASCOT-2 lander.

4. The deep internal structure of the moonlet with the low-frequency radar (LFR).

5. The albedo calibration to improve ground-based observation of other object. This will be accomplished by measuring the albedo with the VIS, TIRI and HFR instruments and comparing to measurements from ground.

The four primary technology research objectives are:

1. Telecommunication Engineering eXperiment (TEX) to qualify and end-to-end deep-space (starting at 0.5 AU) optical link.

2. Moonlet Engineering eXperiment (MEX) to demonstrate aseroid landing and extened operation on it.

3. CubeSat Opportunity Payload Intersatellite Networking Sensors (COPINS) to demonstrate deep-space inter-satellite links.

4. Infra-Red Instrument Navigation Aid (IRINA) to demonstrate the use of an IR instrument potential to support rendezvous phases.

The optical communication system for the spacecraft will not only serve as a first technology demonstration of a deep space optical communication payload, but will also significantly contribute to the research objectives. This is possible as it can be reconfigured in-flight to a high-precision laser altimeter. A description of this mode of operation will be detailed elsewhere.

This paper gives an overview of the AIM mission and presents the design of the laser communication system to be installed on the AIM spacecraft and in ESA's Optical Ground Station (OGS). 


\section{AIM MISSION TIMELINE}

Figure 1 shows the AIM mission timeline with launch in October 2020 and arrival at Didymos in June 2022. After an early characterization phase, several Cubesats and the Mascot lander will be released in August 2022. In August and September a detailed characterization of the binary asteroid system will be performed. In October 2022 the DART impact characterization will take place, which will be followed by post impact monitoring. The mission is scheduled to end in December 2022.

The optical communication link will become operational in July 2022 at distances below 75 Mio km and continue through closest approach of Didymos to Earth of about 12 Mio km in September 2022 until the end of the mission in December 2022.

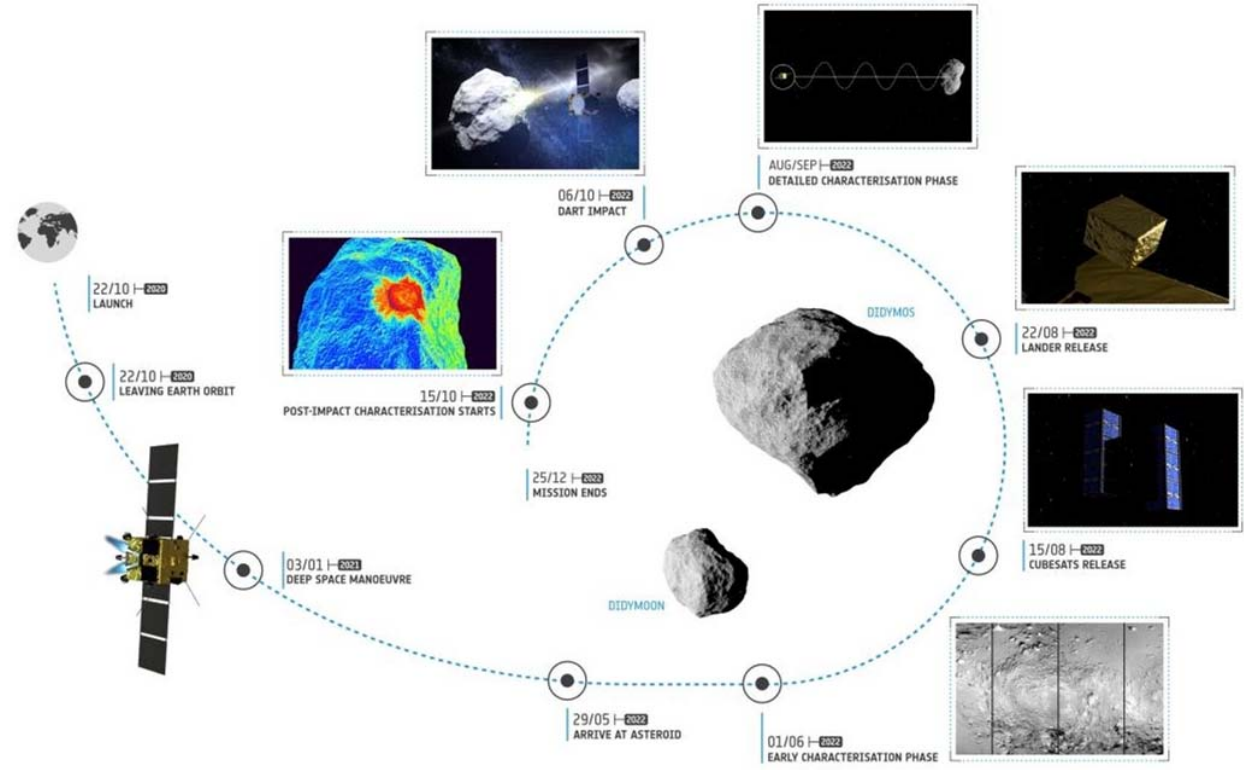

Figure 1 AIM mission timeline.

\section{OPTICAL COMMUNICATION SYSTEM}

As the available development time for the laser communication system is extremely short, equipment from ESA's collaboration with NASA in the Lunar Laser Communication Demonstration (LLCD) experiment is intended to be reused. LLCD was performed in 2013/2014 in collaboration with MIT Lincoln Labs and JPL and these organizations, as well as others, are very welcome to participate in the AIM laser communication experiment by contributing their own optical ground stations and optical communications equipment.

As NASA provided the flight segment for LLCD only hardware for the optical ground station is available, namely the receiver, demodulation, decoding and data storage system. A summary of the optical communication system operational parameters are shown in Table 1.

This paragraph is organized as follows: In Section 0 the design of the laser communication terminal (named OPTEL-D) on board of the AIM spacecraft is introduced and some specific point of interest are highlighted. Next, the optical ground station (OGS) systems are described in Section 3.2 and the link budgets are given in Section 3.3. Section 3.4 describes the receiver and transmitter alignment.

Table 1 Optical Communication Link Parameters.

\begin{tabular}{|l|c|c|c|c|}
\hline & \multicolumn{2}{|c|}{ OGS } & \multicolumn{2}{c|}{ AIM } \\
\hline Parameter & Value & Unit & Value & Unit \\
\hline Geographic longitude: & $16^{\circ} 30^{\prime} 36.36^{\prime}{ }^{\prime}$ & West & NA & \\
\hline Geographic latitude: & $28^{\circ} 17^{\prime} 58.29^{\prime}$ & North & NA & \\
\hline
\end{tabular}




\begin{tabular}{|c|c|c|c|c|}
\hline \multirow[b]{2}{*}{ Parameter } & \multicolumn{2}{|l|}{ OGS } & \multicolumn{2}{|l|}{ AIM } \\
\hline & Value & Unit & Value & Unit \\
\hline Altitude above sea level: & 2393 & $\mathrm{~m}$ & $75-15$ & Mio km \\
\hline Receiver aperture diameter: & 1016 & $\mathrm{~mm}$ & 135 & $\mathrm{~mm}$ \\
\hline Receive wavelength: & 1.55 & $\mu \mathrm{m}$ & 1.064 & $\mu \mathrm{m}$ \\
\hline Receive filter bandwidth: & 5 & $\mathrm{~nm}$ & 5 & $\mathrm{~nm}$ \\
\hline Receiver effective focal length: & 13300 & $\mathrm{~mm}$ & 135 & $\mathrm{~mm}$ \\
\hline Detector/camera pixel diameter: & 200 & $\mu \mathrm{m}$ & 30 & $\mu \mathrm{m}$ \\
\hline Transmit wavelength: & 1064 & $\mathrm{~nm}$ & 1550 & $\mathrm{~nm}$ \\
\hline Transmitter aperture diameter: & 250 & $\mathrm{~mm}$ & 135 & $\mathrm{~mm}$ \\
\hline Transmitter modulation: & $\begin{array}{l}1 / 2 \text { camera frame rate } \\
\text { of the AIM camera }\end{array}$ & & 16 & PPM \\
\hline Transmit data rate: & none & & 195 & kbps \\
\hline
\end{tabular}

\subsection{DESIGN OF THE OPTICAL COMMUNICATION TERMINAL ON AIM (OPTEL-D)}

OPTEL is the name of a family of optical communication terminals designed over the last 15 years by RUAG Space. OPTEL-D, where D stands for deep space, is based on this design heritage and on components that are available to technology readiness level up to TRL6. OPTEL-D's single mirror coarse pointing assembly (CPA) can move in azimuth and (to a limited extend) in elevation direction and is used to sequentially perform communication links with Earth and laser ranging of the asteroid's surface. Figure 2 shows the CPA mounted onto an interface plate connected via three vibration-damping legs (yellow) to a satellite panel. A 135 mm aperture diameter telescope and all optical and electronical components are located on the opposite side of the interface plate covered by a housing and thus inside the spacecraft. The use of an interface plate for the optical head ensures a parallel integration flow of the spacecraft. The alignment of the optical head can be finalized at the OPTEL-D payload integrator without interference with the satellite platform integration.

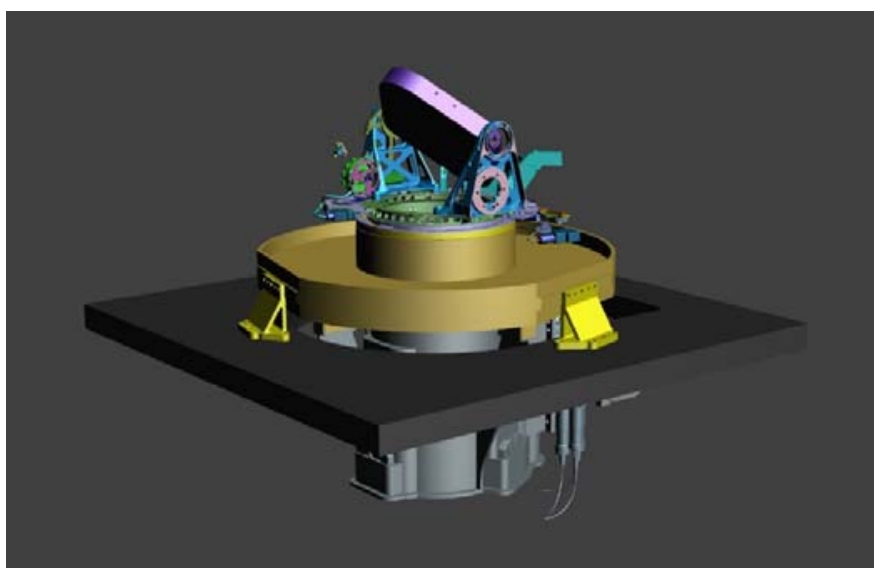

Figure 2 OPTEL-D laser communication terminal schematically mounted on a satellite panel (base plate adapter) with the coarse pointing assembly protruding out of the $\mathrm{S} / \mathrm{C}$ and the optical bench inside the S/C

Figure 3 shows the schematic design of OPTEL-D. The light from an Earth Beacon is received via the coarse pointing assembly (CPA) and an off-axis telescope assembly (TLA) with a free optical diameter of $135 \mathrm{~mm}$. 


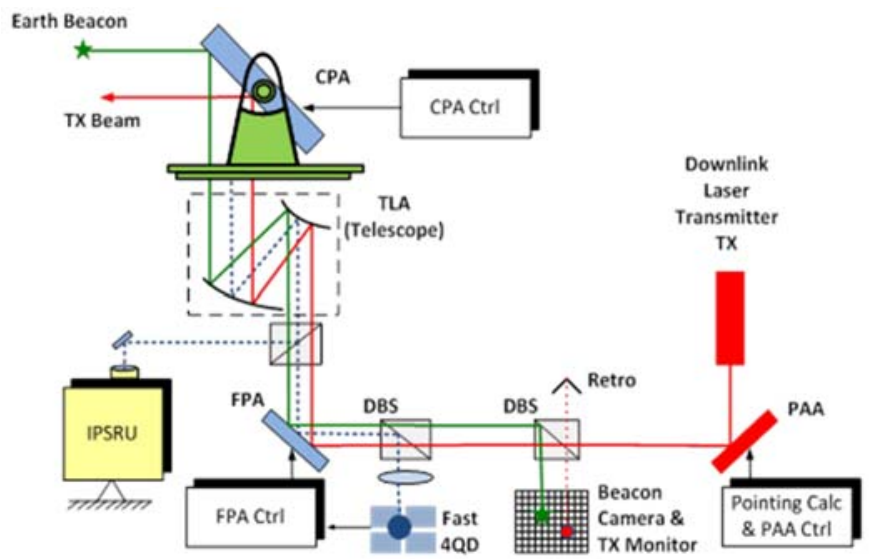

Figure 3 Schematic design of OPTEL-D

The technology readiness level of the TLA is TRL 9 (used in all laser communication terminals of the European Data Relay System, EDRS). The Earth Beacon light may be superimposed with light from an Inertial Pseudo Star Reference Unit (IPSRU). This can be used to cancel out platform vibrations, improving the back-to-earth pointing capabilities of the terminal. The implementation of an IPSRU has not been decided yet. This is a trade-off between payload and platform complexity.

The Earth Beacon is also routed via two Dichroic Beam Splitters (DBS) onto Mercury Cadmium Tellurite (MCT) Beacon Camera and TX Monitor. It provides the pointing, acquisition and tracking functionality and enables calibration of the transmitter and receiver via a Point Ahead Assembly (PAA), a corner-cube retro-reflector (Retro) and the DBS.

The laser data transmitter is routed in the opposite direction via a Point Ahead Assembly (PAA).

A laser ranging functionality (spacecraft to Didymos) will be implemented into OPTEL-D. The currently preferred concept is the use of a circulator inserted into the single-mode output fiber of the power laser transmitter. With the second output of the circulator connected to an InGaAs detector OPTEL-D will be able to simultaneously transmit and receive over link distances below $100 \mathrm{~km}$. A pseudo-random bit sequence will be transmitted towards the target and upon reflection cross-correlated for range determination. Scanning the target with the FPA (or PAA) will produce a three dimensional surface map to support the scientific research objectives.

\subsection{ESA OPTICAL GROUND STATION (OGS)}

The European Space Agency's Optical Ground Station (OGS) is located at the Observatorio del Teide (OT) in the Canary Islands (Tenerife, Spain) shown in Figure 4. Its main purpose is to check out and commission laser communication terminals onboard orbiting spacecraft, i.e. EDRS. In addition the OGS is used for space debris, asteroid and near Earth object detection and for standard astronomical observations.

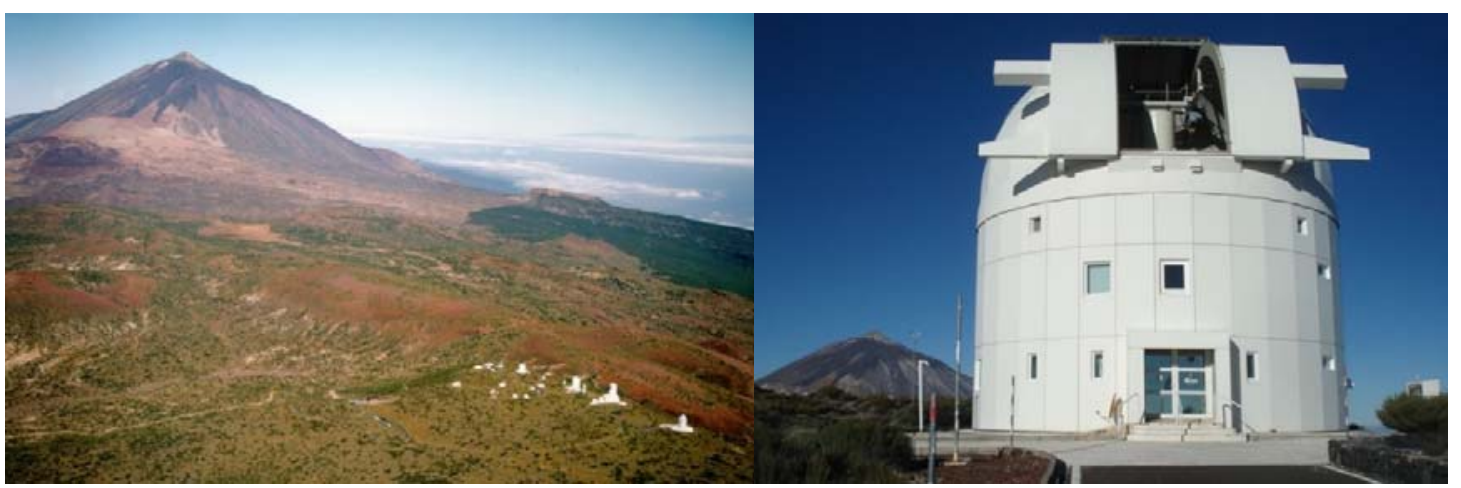

Figure 4 The Observatorio del Teide in Tenerife, Spain (left) and OGS building, which open dome shutter (right).

The Canary island archipelago also offers the opportunity to perform inter-island experiments. From the island of La Palma, at a distance of $142 \mathrm{~km}$, laser and quantum communications experiments are being performed. 
The OGS building houses a $1 \mathrm{~m}$ aperture diameter Zeiss telescope. Current development status of the AIM communication terminal confirms that all objectives for OPTEL-D can be met with the existing $1 \mathrm{~m}$ telescope. For the AIM mission the availability of larger ground based telescopes is being investigated and negotiations are ongoing with the Rohzen National Astronomical Observatory in Bulgaria. Rohzen could make a 2 m Zeiss telescope available and serve as a backup baseline.

The $1 \mathrm{~m}$ sized OGS will also validate the use of larger telescopes for future deep-space optical communication missions. From a link budget point of view the ground telescope diameter scales linearly with link distance and a successful link with the 1 meter diameter OGS telescope over 0.5 AU would therefore validate a 5 m diameter telescope over 2.5 AU (longest distance to Mars) and a $12 \mathrm{~m}$ telescope over 6 AU (longest distance to Jupiter).

Figure 5 (left) shows the equatorial mount of the OGS telescope. It is also called an English mount, because the hour axis (which is parallel to the Earth axis) is supported on both sides, by a (higher) North pillar and a South pillar.

Figure 5 (right) shows the Coudé optical path routing via an evacuated feed-through, which prevents turbulent air exchange and therefore scintillation effects between the telescope floor and the Coudé floor. However, for the AIM mission the Coudé path will be altered by removing the first mirror at the lower right end of the hour axis, such that the Coudé focus will move to the telescope floor inclined by the latitude of the OGS, namely by 28.3 degrees. In this way all refractive elements in the Coudé path are avoided and powerful uplink beacon lasers can be launched from there.

The OGS laser uplink beacon will be intensity modulated at half the camera frame rate on AIM. By subtraction of subsequent image frames the beacon laser can be distinguished from the Earth background and other sources.

Laser downlink communication from the AIM spacecraft will be performed from the stationary Coudé focus.
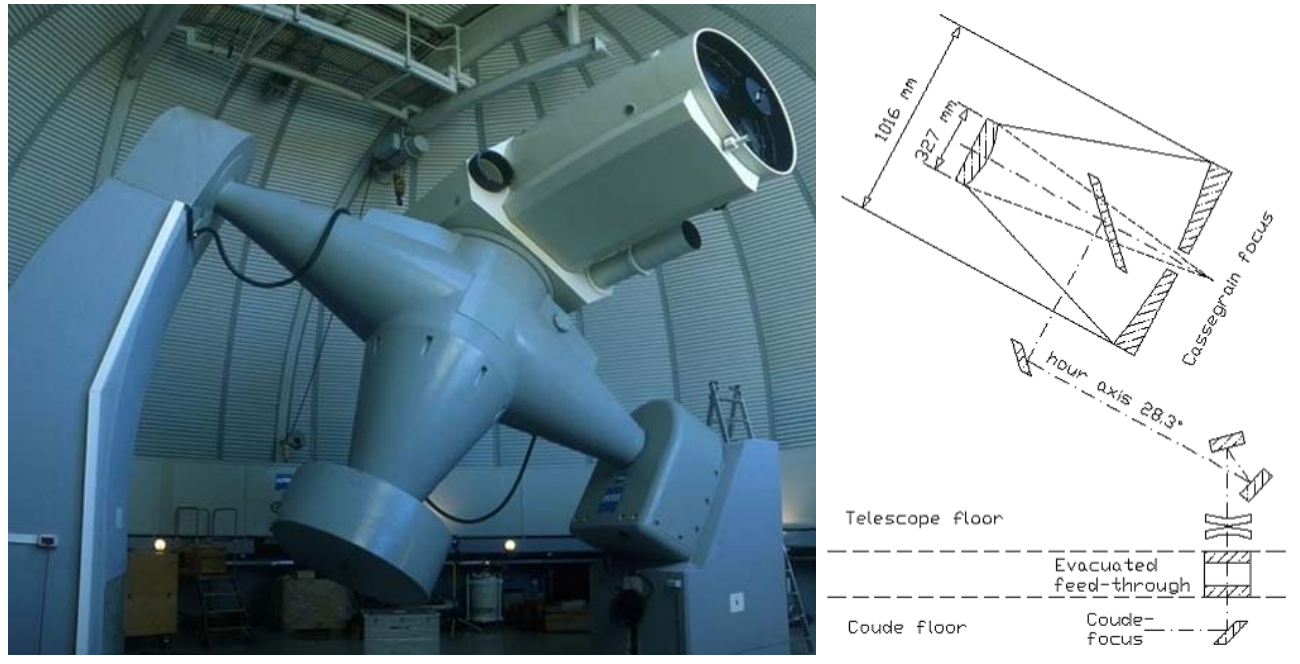

Figure 5 Equatorially mounted $1 \mathrm{~m}$ Zeiss telescope of the OGS (left) and Coudé optical path schematics (right)

The receiver design is based on the development for the Lunar Laser Communication Demonstration (LLCD), where a $1 / 2$-rate serially concatenated pulse-position modulation (SCPPM) turbo like code was implemented. The modulation format is 16-PPM and the user data rate was $38.55 \mathrm{Mbps}$. For the AIM mission the modulation format will stay 16-PPM, but the user data rate is reduced to $195 \mathrm{kbps}$ at the longest link distance of $75 \mathrm{Mio} \mathrm{km}$.

\subsection{LINK BUDGET CALCULATIONS}

Table 2 shows the downlink budget from the AIM spacecraft to the OGS and Table 3 the uplink budget. Both tables indicate the budgets for the maximum and the minimum link distances of 75 Mio km and 15 Mio km respectively.

For the downlink a 3 Watt average power limited amplifier is assumed and the modulation format is 16-PPM. To keep the energy density low, the pulse duration is set to 512 ns. Adding 4 guard slots to the 16-PPM modulation the symbol duration is $10.24 \mu \mathrm{s}$ and the pulse repetition rate $97.7 \mathrm{kHz}$. This results in a raw data rate of $391 \mathrm{kbps}$. With a code rate of 0.5 the effective user data rate becomes $195 \mathrm{kbps}$ from 75 Mio km link distance. During its mission the AIM spacecraft will approach earth further resulting in a reduced free space loss. This gain in received 
photos per laser pulse will be used to increase the data-rate to the Mbps regime. With constant transmitted average power, one can increase the pulse repetition rate and the data rate.

The effective duty cycle of the 16-PPM modulation with guard slots is 0.05 resulting in a modest laser peak power of 100 Watts, which can be handled by space qualified fiber amplifiers.

The detector diameter at the receiving OGS is $200 \mu \mathrm{m}$, covering a field of 3.1 arcsec with a focal length of $13.3 \mathrm{~m}$. The downlink budget is put in relation with the blue-sky brightness (at daytime), however all communication downlinks from Didymos in 2022 will be performed with the OGS in darkness and the blue-sky brightness can be ignored.

Table 2 Downlink Budget from AIM to OGS

\begin{tabular}{|c|c|c|c|}
\hline Parameter & \multicolumn{2}{|l|}{ Value } & Unit \\
\hline \multicolumn{4}{|l|}{ Transmitter } \\
\hline Tx average power (3 Watt): & \multicolumn{2}{|l|}{34.8} & $\mathrm{dBm}$ \\
\hline Tx antenna gain (135 mm): & \multicolumn{2}{|l|}{108.7} & $\mathrm{~dB}$ \\
\hline Tx truncation, WFE and transmission loss (0.63): & \multicolumn{2}{|l|}{-2.2} & $\mathrm{~dB}$ \\
\hline \multicolumn{4}{|l|}{ Channel } \\
\hline Free-space loss (75 - 15 Mio km): & -355.7 & -341.7 & $\mathrm{~dB}$ \\
\hline $\begin{array}{l}\text { Scintillation, mis-pointing, atmospheric absorption and } \\
\text { scattering loss: }\end{array}$ & \multicolumn{2}{|l|}{-3.4} & $\mathrm{~dB}$ \\
\hline \multicolumn{4}{|l|}{ Receiver } \\
\hline Rx antenna gain (1016 mm): & \multicolumn{2}{|l|}{126.3} & $\mathrm{~dB}$ \\
\hline Rx transmission and filter loss (0.5): & \multicolumn{2}{|l|}{-3.0} & $\mathrm{~dB}$ \\
\hline Link margin (0.5) & \multicolumn{2}{|l|}{-3.0} & $\mathrm{~dB}$ \\
\hline Power at receiver $(1.8 \mathrm{pW}-4.5 \mathrm{pW})$ : & -97.5 & -83.5 & $\mathbf{d B m}$ \\
\hline Blue sky brightness (4.2 pW): & \multicolumn{2}{|l|}{-83.8} & $\mathrm{dBm}$ \\
\hline Channel capacity & 391 & 391 & kbps \\
\hline
\end{tabular}
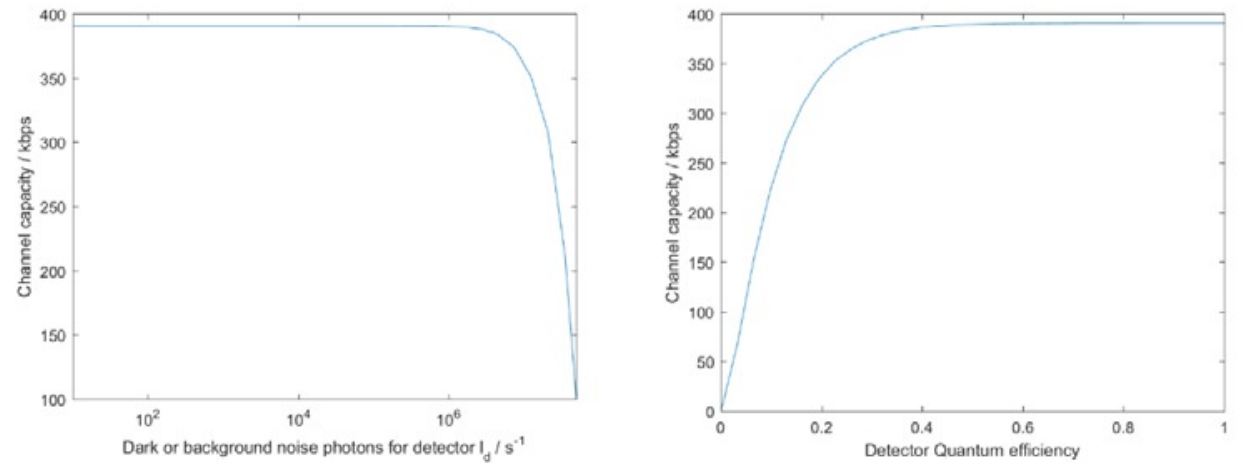

Figure 6 Channel capacity dependence on detector dark noise (left) and quantum efficiency.

With the optical power of $1.8 \mathrm{pW}$ arriving at the detector surface, we expect to achieve data-rates close to the channel capacity by making use of SCPPM coding. According to first simulations based on a Poisson noise dominated, PPM communication channel, we need to ensure a detector noise below $l_{\mathrm{d}}<10^{6}$ with a detector quantum efficiency of $Q E>0.5$ (s. Figure 6).

In Table 3 the uplink budget is calculated for four $600 \mathrm{~W}$ laser beacon sources transmitted from the OGS at wavelengths of $1064 \mathrm{~nm}$. The transmit apertures are selected as $250 \mathrm{~mm}$. This leads a beam divergence of the 
laser beacon ( $35.3 \mu \mathrm{rad}$ at $1 / \mathrm{e}^{2}$ ), which needs to be aligned to the receive path and to calibration stars with an accuracy at least five times better.

A $2.4 \mathrm{~kW}$ beacon needs to be launched from multiple sub-apertures (e.g. four) of the OGS telescope in order to reduce the power density of the optical surfaces. This can be done by using four individual lasers or with a single laser split into four beams. The four laser solution has the advantage of operational redundancy and of a more homogenous far-field illumination pattern caused by the superposition of four laser beams. A single laser solution would generate stronger interference effects (fades and surges) in the far field caused by beam interference due to the coherence length of the laser.

Table 3 Uplink Budget from OGS to AIM per detector pixel

\begin{tabular}{|c|c|c|c|}
\hline & \multicolumn{3}{|c|}{$1064 \mathrm{~nm}$} \\
\hline Parameter & \multicolumn{2}{|c|}{ Value } & Unit \\
\hline \multicolumn{4}{|l|}{ Transmitter } \\
\hline Tx average power $(4 \mathrm{x} 600 \mathrm{~W}=2400 \mathrm{~W})$ : & & 63.8 & $\mathrm{dBm}$ \\
\hline Tx antenna gain $(250 \mathrm{~mm})$ : & & 98.1 & $\mathrm{~dB}$ \\
\hline Co-alignment pointing loss (0.9) & & -0.5 & $\mathrm{~dB}$ \\
\hline Tx transmission loss $(0.8)$ : & & -1.0 & $\mathrm{~dB}$ \\
\hline \multicolumn{4}{|l|}{ Channel } \\
\hline Free-space loss (75 - 15 Mio km): & -359.0 & -345.0 & $\mathrm{~dB}$ \\
\hline Atmospheric absorption and scattering loss: & & -8.2 & $\mathrm{~dB}$ \\
\hline \multicolumn{4}{|l|}{ Receiver } \\
\hline Rx antenna gain (135 mm): & & 112.0 & $\mathrm{~dB}$ \\
\hline Rx transmission loss inclusive filter (0.7): & & -1.6 & $\mathrm{~dB}$ \\
\hline Power at receiver $(0.2 \mathrm{pW}-5.9 \mathrm{pW})$ : & -96.3 & -82.3 & dBm \\
\hline Earth background (1.9 pW): & & -87.14 & $\mathrm{dBm}$ \\
\hline
\end{tabular}

The uplink budget for the beacon laser is presented in Table 3. At longest link distance, the earth background outshines the received optical power by almost $10 \mathrm{~dB}$. Therefore, a slow modulation of the uplink laser is used to distinguish between background and signal photons.

\subsection{OGS RECEIVER/TRANSMITTER DESIGN AND ALIGNMENT}

The transmit laser is coupled into the telescope’s Coudé path via a dichroic beam splitter (a glass plate that reflects the wavelength spectrum above $1.2 \mu \mathrm{m}$ and transmits the wavelength spectrum below $1.2 \mu \mathrm{m}$ ), whose reflection is optimized for $1.06 \mu \mathrm{m}$ and transmission for $1.55 \mu \mathrm{m}$.

For alignment of the optical axes of transmitter and receiver, a high precision ( $<1$ arcsec) corner-cube retroreflector (CCRR) with a diameter of $120 \mathrm{~mm}$ is mounted at the $1 \mathrm{~m}$ aperture of the OGS telescope. A weak $1550 \mathrm{~nm}$ pilot laser beam, co-aligned to and emitted from the transmit laser, is then retro-reflected through the telescope and the beam splitter towards the four quadrant detector.

\section{CONCLUSIONS}

The technical design challenges of the optical communication system for the Asteroid Impact Mission (AIM) have been presented. Despite a link distance of "only” 75 Mio km, which is short in comparison to planetary distances, it was found that acquisition of the laser beams from the space terminal and from the ground terminal are the most challenging issues. The use of a modulated continuous wave beacon laser turned out to be the most efficient implementation, allowing the space terminal to target the ground terminal with sufficient precision.

It is hoped that the Asteroid Impact Mission (AIM) will become the first demonstration of a deep-space optical communication link. 


\section{ACKNOWLEDGMENT}

The authors would like to thank Igor Zayer, Robert Daddato and Klaus-Jürgen Schulz from ESOC for helpful discussions.

\section{REFERENCES}

[1] Asteroid Impact Mission, Payload Assessment (AIM 3P), CDF Integrated Design Model (IDM), C 2004 2014 by ESA.

[2] Z. Sodnik, B. Furch B, H. Lutz, “The ESA Optical Ground Station - Ten Years Since First Light”, ESA bulletin 132, November 2007, pp. $34-40$.

[3] T. Jono, Y. Takayama, N. Kura, K. Ohinata, Y. Koyama, K. Shiratama, Z. Sodnik, B. Demelenne, A. Bird, and K. Arai, “OICETS on-orbit laser communication experiments,” Proc. SPIE, 2006, vol. 6105, pp. 1323.

[4] R. Lange R., B. Smutny, “Homodyne BPSK-based optical inter-satellite communication links”, Proc. SPIE, 2007, Vol. 6457, p. 645703.

[5] K.-J. Schulz, J. Rush et al., “Optical Link Study Group Final Report,” IOAG-15b, June 2012.

[6] LLCD Lunar Lasercom Space Terminal (LLST) to Co-operating Ground Terminal (CGT) Interface Control Document, LLCD-CGT-ICD-001, Goddard Space Flight Center.

[7] F. Arnold, M. Mosberger, J. Widmer and F. Gambarara, "Ground receiver unit for optical communication between LADEE spacecraft and ESA ground station”, SPIE, 2014, vol. 8971

[8] M. Sans, Z. Sodnik, I. Zayer and R. Daddato, “Design of ESA’s Optical Ground Station for Participation in LLCD”, Proc. ICSOS 2012, Ajaccio

[9] B.S. Robinson, D.M. Boroson, D.A. Burianek, D.V. Murphy, “The Lunar Laser Communications Demonstration,” Space Optical Systems and Applications (ICSOS), 2011 International Conference on , pp.54-57, 11-13 May 2011.

[10] Z. Sodnik, H. Smit, M. Sans, I. Zayer, M. Lanucara, I. Montilla, A. Alonso, "LLCD Operations using the Lunar Lasercom OGS Terminal”, SPIE, 2014, vol. 8971 